University of Wollongong

Research Online

Faculty of Engineering - Papers (Archive)

Faculty of Engineering and Information

Sciences

2004

\title{
The electron energy spectrum and thermionic device efficiency
}

Mark F. O'Dwyer

University of Wollongong, markod@uow.edu.au

T. E. Humphrey

University of Wollongong

R. A. Lewis

University of Wollongong, roger@uow.edu.au

C. Zhang

University of Wollongong, czhang@uow.edu.au

Follow this and additional works at: https://ro.uow.edu.au/engpapers

Part of the Engineering Commons

https://ro.uow.edu.au/engpapers/5586

\section{Recommended Citation}

O'Dwyer, Mark F.; Humphrey, T. E.; Lewis, R. A.; and Zhang, C.: The electron energy spectrum and thermionic device efficiency 2004.

https://ro.uow.edu.au/engpapers/5586

Research Online is the open access institutional repository for the University of Wollongong. For further information contact the UOW Library: research-pubs@uow.edu.au 


\title{
The Electron Energy Spectrum and Thermionic Device Efficiency
}

\author{
M. F. O’Dwyer, T. E. Humphrey, R. A. Lewis, C. Zhang \\ School of Engineering Physics \\ University of Wollongong, Wollongong, NSW 2522, Australia \\ Email:mo15@uow.edu.au
}

\begin{abstract}
The influence of the electron energy spectrum of solid-state thermionic devices on electronic efficiency is analyzed. Calculations are performed on both single and multibarrier GaAs/AIGaAs and InGaAs/InAlAs systems. Analysis reveals a wide barrier is desirable for single-barrier thermionic devices due to the associated sharpness in the electron energy spectrum. It is also shown that high electronic efficiency may be achieved in multibarrier thermionic devices consisting of thin barriers, which would separately give low efficiency, but together can be arranged to produce a desirable electron energy spectrum.
\end{abstract}

\section{INTRODUCTION}

Traditional vacuum thermionic power generators with macroscopic gaps between emitter and collector plates are generally limited to very high temperatures $\left(T_{H}>1000 \mathrm{~K}\right)$ [1]-[3]. Refrigeration using vacuum-based thermionic devices was suggested by Mahan [4], however, these too are limited to high temperature applications due to the low-work-function $(<0.3 \mathrm{eV})$ emitter materials required for low-temperature applications.

It was suggested by Shakouri and Bowers that semiconductor nanostructures could be employed to achieve the low barrier heights desired [5], [6]. Mahan et al. later proposed multilayer structures as a way of reducing phonon heat leaks inherent in the use of solid-state rather than vacuum-based devices, thus improving efficiency [7], [8]. Successful solidstate thermionic cooling has been reported by Shakouri et al. using a single-barrier InGaAsP based structure [9] and by LaBounty et al. using a 25-barrier InGaAsP based multilayer system [10]. In both cases the cooling achieved was about one degree.

Such solid-state nanostructures offer the possibility of engineering the energy spectrum of electrons transmitted between the collector and emitter in a manner which was not previously possible with vacuum-based technology. In light of this new design freedom, it is useful to examine the effect that the electron energy spectrum has on the 'electronic efficiency' of thermionic devices, which is the efficiency due to electronic processes only [11]. An improvement in electronic efficiency at constant power will improve the overall efficiency of a practical thermionic device in which phonon, radiative and lead effects are also important.

In this paper both single and multibarrier GaAs/AlGaAs and InGaAs/InAlAs systems are analyzed. It is shown that wide barriers are desirable in a single-barrier device due to the sharpness of the electron energy spectrum. A particular width exists at which the further increase in the width and associated sharpness does not produce any appreciable improvement in the electronic efficiency. It is also shown that multiple thin barriers, which would have low efficiency when used alone, may be arranged so that the electron energy spectrum rises sharply and as a result higher electronic efficiency is achieved. These results will be relevant to device design where multiple barriers are present over lengths less than the electron mean free path.

\section{BALlistic TRANSPORT THEORY}

A solid-state thermionic device consists of two electron reservoirs separated by a barrier, or series of barriers, which serve to filter the flow of electrons between the reservoirs. Electrons may travel ballistically over the potential barriers due to an applied thermal and electrochemical potential (voltage) difference. At low applied bias voltages, the system will behave as a heat engine producing electrical power. At higher bias voltages the net current reverses direction and the device behaves as a refrigerator extracting heat. In a semiconductorbased device that is translationally invariant in the $y$ and $z$ directions the energy (or more precisely, momentum) is filtered in the direction of transport, $x$, only. We therefore denote such systems as ' $k_{x}$ filtered thermionic devices'.

In a device where the electron mean free path between inelastic collisions is greater than the width of the barrier or system of barriers the electron current may be calculated based on the transmission probability of the barrier system. In a $k_{x}$ filtered thermionic device the transmission probability is a function of what may be loosely defined as the kinetic energy in the $x$ direction, $E_{x}=\hbar^{2} k_{x}^{2} / 2 m^{*}$, and the net electrical current flowing between the cold and hot reservoirs is given by

$$
J_{e}=e \int_{U_{C}}^{\infty}\left[N^{x}\left(E_{x}, T_{C}, \mu_{C}\right)-N^{x}\left(E_{x}, T_{H}, \mu_{H}\right)\right] \tau\left(E_{x}\right) d E_{x},
$$

where

$N^{x}\left(E_{x}, T_{C / H}, \mu_{C / H}\right)=\frac{m^{*} k_{B} T_{C / H}}{2 \pi^{2} \hbar^{3}} \ln \left[1+\exp \left(-\frac{E_{x}-\mu_{C / H}}{k_{B} T_{C / H}}\right)\right]$

is the number of electrons with kinetic energy in the $x$ direction $E_{x}$ arriving at the reservoir interface per unit area per unit time, $U_{C}$ is the bottom energy of the cold reservoir, $\tau\left(E_{x}\right)$ is the transmission probability, $m^{*}$ is the effective mass of electrons 
and $T_{C / H}$ and $\mu_{C / H}$ are temperatures and electrochemical potentials of the cold/hot reservoirs respectively. It is assumed that the cold reservoir electrochemical potential is greater than that of the hot reservoir.

The heat current flowing between the reservoirs may be calculated by considering the energy carried by each electron. The energy component in the $x$ direction is filtered and given by $E_{x}$, however, the energies in the $y$ and $z$ directions may take any value. Assuming Maxwell-Boltzmann statistics, the average energy contribution in each of these degrees of freedom is $k_{B} T / 2$. The energy removed/added by an electron is given by the difference between the electron energy and electrochemical potential of the reservoir. The net heat current out of the cold/hot reservoir is therefore given by

$$
\begin{gathered}
J_{q}^{C / H}= \pm \int_{U_{C}}^{\infty}\left[\left(E_{x}+k_{B} T_{C}-\mu_{C / H}\right) N^{x}\left(E_{x}, T_{C}, \mu_{C}\right)\right. \\
\left.-\left(E_{x}+k_{B} T_{H}-\mu_{C / H}\right) N^{x}\left(E_{x}, T_{H}, \mu_{H}\right)\right] \tau\left(E_{x}\right) d E_{x} .
\end{gathered}
$$

The electronic efficiency of the system as a heat engine and the coefficient of performance (COP) as a refrigerator are given by

$$
\eta_{H E}=V J_{e} / J_{q}^{H}
$$

and

$$
\eta_{R}=J_{q}^{C} / V J_{e}
$$

respectively under applied bias $V$.

The transmission probability is calculated by obtaining a numerical solution to the time-independent Schrödinger equation based on Airy functions [12], [13]. In this paper the electron effective mass is assumed to be constant throughout the device.

\section{Single-BARrier Thermionic Device}

We begin by analyzing the electron energy spectra and efficiencies/COPs associated with single-barrier devices. We first calculate the electronic efficiency of a GaAs/AlGaAs system with effective mass of $0.067 m_{e}$ and barrier height $0.3 \mathrm{eV}$ [12]. The barriers in this system are chosen to be angular in shape as shown in Figure 1(a), corresponding to the traditional theoretical conception of a potential barrier. An InGaAs/InAlAs system is also analyzed with effective mass of $0.041 m_{e}$ and barrier height of $0.5 \mathrm{eV}$ [14]. In this instance the system is modeled with a rounded barrier, shown in Figure 1(b), which may more closely represent the potential of a realistic device. The width of both barriers were then varied and the performance of the systems calculated.

As the bias on the single-barrier is increased, system behavior gradually changes. At low applied bias the system behaves as a heat engine, as shown in Figure 2 for the 30$\mathrm{nm}$ GaAs/AlGaAs system. At a certain applied bias the net heat current switches direction and flows from the cold to hot reservoir and the system begins to act as a refrigerator. Figure 2 shows that clear optimal biases exists for maximum heat engine efficiency and refrigerator COP. Similar figures may be generated for each of the systems and each may have a different bias where maximum efficiency and COP are achieved. The values presented for each system are the

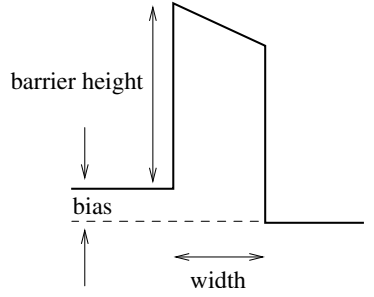

(a) Single Angular Barrier

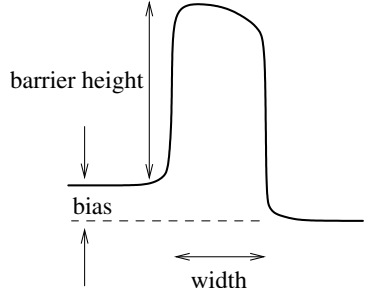

(b) Single Rounded Barrier

Fig. 1. Single potential barrier profiles

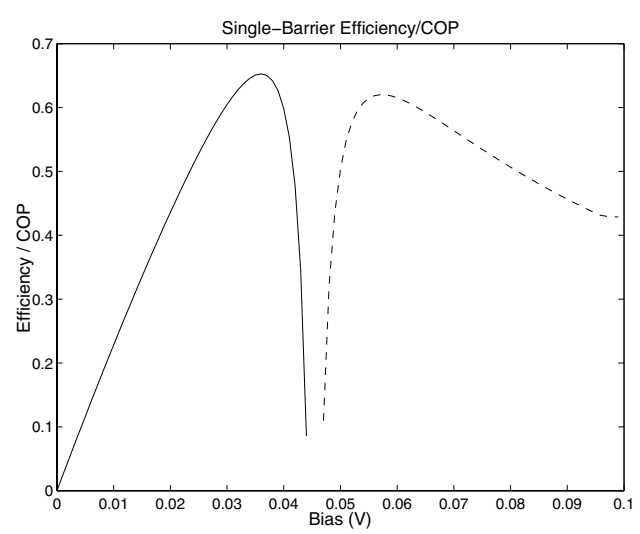

Fig. 2. Efficiency (solid line) and COP (dashed line) of a 30-nm single-barrier InGaAs/InAlAs system.

maximum efficiency/COP obtained by tuning the system bias in this way from 0 to $0.1 \mathrm{~V}$. The systems were chosen to have a cold-side electrochemical potential of $0.1 \mathrm{eV}$ and cold/hot reservoir temperatures of 270/300 K.

Table I shows the calculated maximum efficency/COP for a variety of single-barrier widths relative to the Carnot value. A wider barrier produces a transmission probability that rises more sharply, which is desirable in terms of device efficiency. Figure 3 shows the transmission probability associated with the 10-nm and 100-nm InGaAs/InAlAs single-barrier systems and the significant difference between the sharpness of their energy spectra. This is reflected in the results shown in

TABLE I

SINGLE-BARRIER EFFICIENCY/COP RELATIVE TO THE CARNOT VALUE

\begin{tabular}{|c|c|c||c|c|c|}
\multicolumn{4}{c}{ GaAs/AlGaAs } & \multicolumn{3}{c|}{ InGaAs/InAlAs } \\
\hline Width (nm) & $\eta_{H E}^{\max }$ & $\eta_{R}^{\max }$ & Width $(\mathrm{nm})$ & $\eta_{H E}^{\max }$ & $\eta_{R}^{\max }$ \\
\hline 10 & 0.43 & 0.39 & 10 & 0.33 & 0.28 \\
12 & 0.63 & 0.60 & 25 & 0.36 & 0.32 \\
13 & 0.70 & 0.67 & 27.5 & 0.50 & 0.46 \\
15 & 0.75 & 0.73 & 30 & 0.65 & 0.62 \\
20 & 0.77 & 0.75 & 40 & 0.84 & 0.83 \\
25 & 0.77 & 0.75 & 50 & 0.86 & 0.84 \\
40 & 0.77 & 0.75 & 100 & 0.87 & 0.85 \\
\hline
\end{tabular}




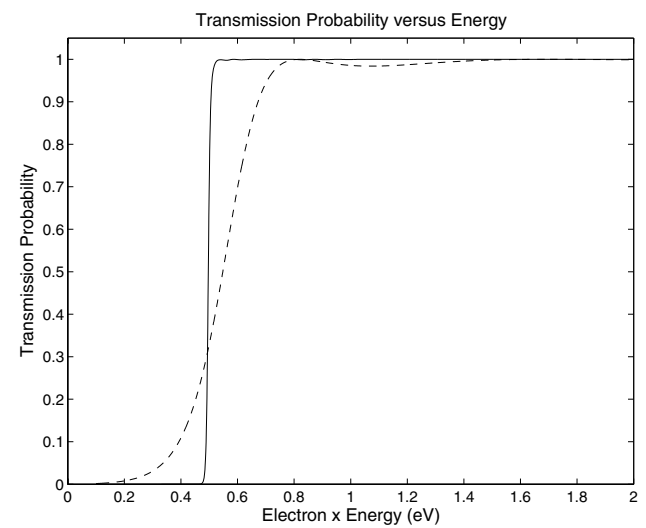

Fig. 3. Transmission probability of 10-nm (dashed line) and 100-nm (solid line) single-barrier InGaAs/InAlAs thermionic devices under no applied bias.

Table I, with narrow barriers having low efficiency and wider barriers higher efficiency. The primary difference between the angular $\mathrm{GaAs} / \mathrm{AlGaAs}$ and rounded $\mathrm{InGaAs} / \mathrm{InAlAs}$ barrier transmission probabilities (apart from the obvious difference in the cutoff energy associated with barrier height) is the absence of the oscillatory phenomena for energies above the barrier height for the latter, as shown in Figure 4. The oscillations may result in an slight increase in the electronic efficiency due to reduced non-ideal electron energy contribution, however, such phenomena is unlikely to be as pronounced in a real device. Neglecting other considerations such as phonon scattering, a wide barrier is also preferable since the lattice heat leaked is inversely proportional to barrier width. In both instances efficiency reaches a maximum value at a certain width beyond which it remains roughly constant. Therefore, if barrier width is an issue there is no benefit from an electronic efficiency standpoint to increase barrier width beyond this value. This result, that wider barriers are preferable, is consistent with the conventional view in solid-state thermionics that the barriers should be wide so as to minimize tunneling current or remove hot electrons only. The barrier width must not exceed the electron mean free path however as transit must be ballistic.

\section{Multibarrier Thermionic Devices}

We now extend our analysis to multibarrier thermionic devices where the electron mean free path in the device is less than or equal to the length of the device. This limits the analysis to structures with thin barriers which have been shown to have relatively poorer efficiencies in Section III. However, it will be shown that high electronic efficiency may be achieved using thin barriers in a multilayer structure which used separately would have low electronic efficiency. Multibarrier structures are generally employed to reduce lattice thermal conductivity. It will be shown here that they may also be used to improve electronic efficiency, which, when combined with reduced lattice conductivity, may result in a considerably superior device.

In such multibarrier devices the electron energy spectrum is more complex than in single-barrier devices. It has been

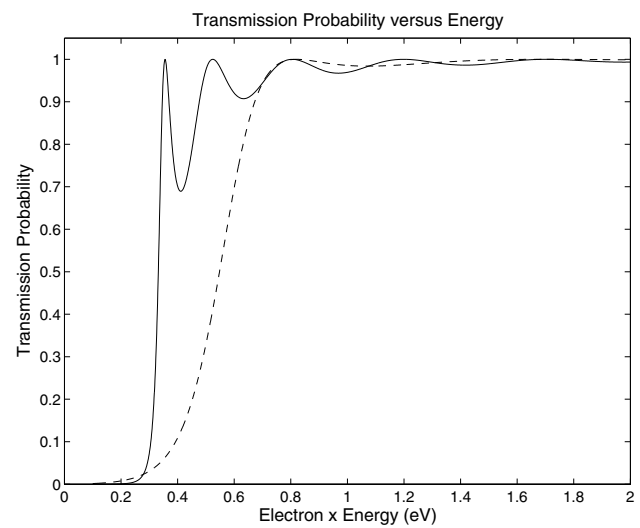

Fig. 4. Transmission probability of 10-nm wide angular GaAs/AlGaAs (solid line) and rounded InGaAs/InAlAs (dashed line) systems under no applied bias.

shown from a theoretical point of view, in terms of electronic efficiency, that the ideal filter for a thermionic device is one which transmits electrons of only a single energy [15]. While it is not currently possible to develop devices which have this ideal filtering, it is possible to design a device such that a sharp peak occurs in the transmission probability. Here fourbarrier angular GaAs/AlGaAs and rounded InGaAs/InAlAs systems will be analyzed where the structures have been chosen to produce a sharp peak in the energy spectrum which is conducive to high electronic efficiency.

The transmission probability for the four-barrier $\mathrm{GaAs} / \mathrm{AlGaAs}$ system under ideal refrigerator bias is shown in Figure 5. The transmission probability for the fourbarrier InGaAs/InAlAs system exhibits similar phenomena. The rise in the electron energy spectrum on the low energy side is much sharper here than in a $10-\mathrm{nm}$ single-barrier device shown in Figure 4 and improves the electronic efficiency significantly. Further, the reduction in the number of electrons with energy greater than the ideal value will also slightly increase electronic efficiency (at the expense of power, however).

Figure 6 shows the efficiency and COP versus applied bias for the four-barrier GaAs/AlGaAs system. This behavior is more erratic than shown that in Figure 2 for the single-barrier system due to the fine structure in the electron spectrum in this case. There are still clear maximum efficiency and COP values which may be found by tuning the bias as before. The maximum relative electronic efficiency and COP calculated for the four angular barrier GaAs/AlGaAs system were both 0.75 . This is remarkably high and is comparable to the maximum efficiency/COP achieved with wide single barriers, even though in this case narrow barriers have been used, which gave low efficiency in single-barrier systems.

For the four rounded barrier InGaAs/InAlAs system the maximum relative electronic efficiency was calculated to be 0.54 and maximum relative $\mathrm{COP}$ was 0.49 . Again these values are significantly higher than those achieved with a single barrier of the same width, however, are significantly below 


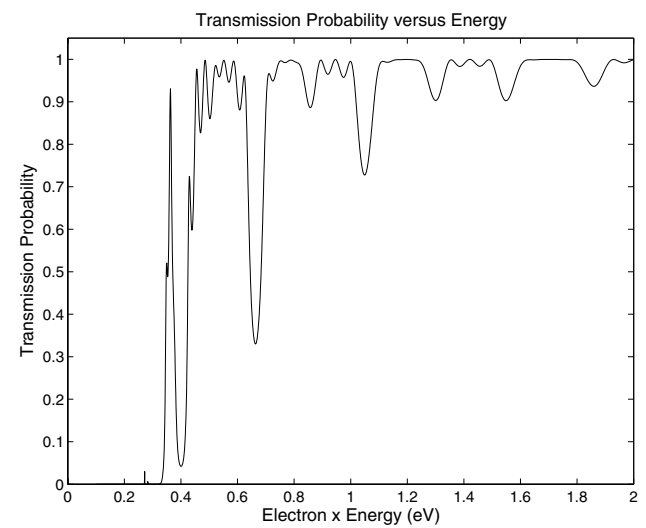

Fig. 5. Transmission probability for a four angular barrier GaAs/AlGaAs system with barrier width $10 \mathrm{~nm}$ and barrier separation of $10 \mathrm{~nm}$ under optimum refrigerator bias.

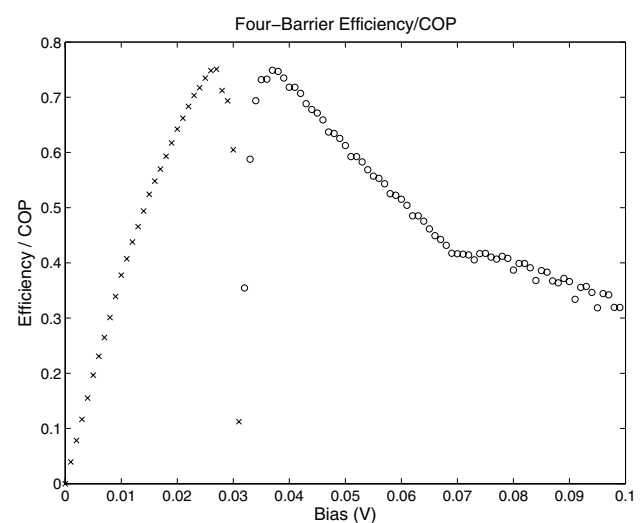

Fig. 6. Efficiency (crosses) and COP (circles) for a four angular barrier GaAs/AlGaAs system with barrier width $10 \mathrm{~nm}$ and separation of $10 \mathrm{~nm}$ as functions of applied bias.

the maximum values achieved with wide single barriers.

Clearly with careful multibarrier barrier arrangement, an energy spectrum may be engineered to achieve higher efficiency than would be possible with equivalent individual barriers.

\section{CONCLUSIONS}

It has been shown that from the perspective of electronic efficiency, devices should be designed so that the electron energy spectrum rises sharply. For both single angular barrier GaAs/AlGaAs systems and single rounded barrier In$\mathrm{GaAs} / \mathrm{InAlAs}$ systems it has been shown that a sharp electron energy spectrum and high electronic efficiency may be achieved with a wide barrier. This is consistent with the conventional view in solid-state thermionics that the barriers should be sufficiently wide so that tunneling current is negligible [16]. Beyond a certain barrier width, however, increasing the width does not increase the sharpness of the electron energy spectrum appreciably enough to make a significant difference to the electronic efficiency.

Multibarrier thermionic devices have been analyzed where the electron mean free path was approaching the length of the device. It has been shown that thin barriers, which would otherwise produce low electronic efficiency if used alone, could be arranged in such a way that high electronic efficiency is achieved. This suggests that barriers in multilayer devices do not necessarily need to be wide for high electronic efficiency. Thus, one may utilize the fact that electrons may 'feel' the influence of more than one barrier when considering the transmission of electrons in the system to engineer a desirable electron energy spectrum. A more complex model would be required to analyze the performance of a device whose overall length is significantly greater than the electron mean free path where the barriers are thin enough so that an electrons transmission through the system depends on more than one barrier at any one time. However, based on the analysis presented here, it would appear that in such devices high electronic efficiency may be achieved by considering the electron energy spectrum due to more than one barrier. Thus, the use of multiple barriers over lengths less than the mean free path may inherently increase the electronic efficiency in many cases, effectively 'for free'. Since thin barriers may be preferable for other reasons, for example to enhance phonon scattering in a particular structure, the influence multiple barriers have on the electron energy spectrum may be of significant benefit to device efficiency.

\section{REFERENCES}

[1] W. Schilichter, Ph.D. dissertation, University of Gottingen, 1915.

[2] J. M. Houston, "Theoretical efficiency of the thermionic energy converter," J. Appl. Phys., vol. 30, no. 4, pp. 481-487, 1958

[3] V. C. Wilson, "Conversion of heat to electricity by thermionic emission," J. Appl. Phys., vol. 30, no. 4, pp. 475-481, 1958.

[4] G. D. Mahan, "Thermionic refrigeration," J. Appl. Phys., vol. 76, no. 7 , pp. 4362-4366, 1994.

[5] A. Shakouri and J. E. Bowers, "Heterostructure integrated thermionic coolers," Appl. Phys. Lett., vol. 71, no. 9, pp. 1234-1236, 1997.

[6] A. Shakouri and J. E. Bowers, "Heterostructure integrated thermionic refrigeration," 16th Conf. Thermoelectrics, pp. 636-340, 1997.

[7] G. D. Mahan and L. M. Woods, "Multilayer thermionic refrigeration," Phys. Rev. Lett., vol. 80, no. 18, pp. 4016-4018, 1998.

[8] G. D. Mahan, J. O. Sofo, and M. Bartkowiak, "Multilayer thermionic refrigerator and generator," J. Appl. Phys., vol. 83, no. 9, pp. 4683-4689, 1998.

[9] A. Shakouri, C. LaBounty, J. Piprek, P. Abraham, and J. E. Bowers, "Thermionic emission cooling in single barrier heterostructures," Appl. Phys. Lett., vol. 74, no. 1, pp. 88-89, 1999.

[10] C. Labounty, A. Shakouri, P. Abraham, and J. E. Bowers, "Integrated cooling for optoelectronic devices," Proc. SPIE, vol. 3950, pp. 69-75, 2000 .

[11] G. N. Hatsopoulos and E. P. Syftopoulos, Thermionic Energy Conversion Volume I: Processes and Devices. London: The MIT Press, 1973.

[12] J. H. Davies, The Physics of Low Dimensional Semiconductors - An Introduction, Cambridge, 1998.

[13] K. F. Brennan and C. J. Summers, "Theory of resonant tunneling in a variably spaced multiquantum well structure: An Airy function approach," J. Appl. Phys., vol. 61, no. 2, pp. 614-623, 1987.

[14] D. Vashaee and A. Shakouri, "Electronic and thermoelectric transport in semiconductor and metallic superlattices," J. Appl. Phys., vol. 95, no. 3, pp. 1233-1245, 2004.

[15] T. E. Humphrey, R. Newbury, R. P. Taylor, and H. Linke, "Reversible quantum Brownian heat engines for electrons," Phys. Rev. Lett., vol. 89 no. 11 , pp. $116801-1-4,2002$.

[16] B. Lough, S. P. Lee, R. A. Lewis, and C. Zhang, "Electronic thermal transport and thermionic cooling in semiconductor multi-quantum-well structures," Comp. Phys. Comm., vol. 142, pp. 274-280, 2001. 